OPEN ACCESS

Edited by: Shamim Hossain, Kyushu University, Japan

Reviewed by:

Genny Orso

University of Padua, Italy

Giuseppe Calamita,

University of Bari Aldo Moro, Italy

*Correspondence: Bing Tian

tianbing@zju.edu.cn

Specialty section:

This article was submitted to

Cellular Biochemistry,

a section of the journal

Frontiers in Cell and Developmental

Biology

Received: 07 May 2020

Accepted: 21 July 2020

Published: 31 August 2020

Citation:

Zhou Y, Yu N, Zhao J, Xie Z Yang Z and Tian B (2020) Advances in the Biosynthetic Pathways and Application Potential

of Plasmalogens in Medicine.

Front. Cell Dev. Biol. 8:765.

doi: 10.3389/fcell.2020.00765

\section{Advances in the Biosynthetic Pathways and Application Potential of Plasmalogens in Medicine}

\author{
Yulong Zhou ${ }^{1,2}$, Ning Yu', Jie Zhao ${ }^{3}$, Zhenming Xie ${ }^{2}$, Zhaonan Yang ${ }^{1,2}$ and Bing Tian ${ }^{2 *}$ \\ ${ }^{1}$ Key Laboratory for Green Processing of Chemical Engineering of Xinjiang Bingtuan, School of Chemistry and Chemical \\ Engineering, Shihezi University, Shihezi, China, ${ }^{2}$ MOE Key Laboratory of Biosystem Homeostasis and Protection, College \\ of Life Sciences, Zhejiang University, Hangzhou, China, ${ }^{3}$ Department of Applied Biological Science, Zhejiang University, \\ Hangzhou, China
}

Plasmalogens are a special class of polar glycerolipids containing a vinyl-ether bond and an ester bond at sn-1 and sn-2 positions of the glycerol backbone, respectively. In animals, impaired biosynthesis and regulation of plasmalogens may lead to certain neurological and metabolic diseases. Plasmalogens deficiency was proposed to be strongly associated with neurodegenerative and metabolic diseases, such as Alzheimer's disease (AD) and Parkinson's disease (PD), and appropriate supplement of plasmalogens could help to prevent and possibly provide therapy of these diseases. Plasmalogens evolved first in anaerobic bacteria with an anaerobic biosynthetic pathway. Later, an oxygen-dependent biosynthesis of plasmalogens appeared in animal cells. This review summarizes and updates current knowledge of anaerobic and aerobic pathways of plasmalogens biosynthesis, including the enzymes involved, steps and aspects of the regulation of these processes. Strategies for increasing the expression of plasmalogen synthetic genes using synthetic biology techniques under specific conditions are discussed. Deep understanding of plasmalogens biosynthesis will provide the bases for the use of plasmalogens and their precursors as potential therapeutic regimens for age-related degenerative and metabolic diseases.

Keywords: plasmalogens, biosynthesis, anaerobic, oxygen-dependent, aging disease

\section{INTRODUCTION}

Plasmalogens (1-O-alk-1'-enyl 2-acyl glycerol phospholipids and glycolipids), also called plasmenyl phospholipid and plasmenyl glycolipids, are a special group of polar lipids, accounting for approximately $18-20 \mathrm{~mol} \%$ of the total phospholipids in cell membranes of almost all mammalian. They are the constituents of biomembranes, which has a diversity of functions such as cell homeostasis, signaling and neural transmission (Ferlay et al., 2015; Dean and Lodhi, 2018). Plasmalogen contains a vinyl ether $(-\mathrm{O}-\mathrm{CH}=\mathrm{CH}-)$-linked chain at sn-1 position and an ester chain at sn-2 position of glycerol backbone (Snyder, 1999; Braverman and Moser, 2012), respectively (Figure 1). Plasmalogens in animal tissues usually have a polyunsaturated acyl chain at the sn-2 position. Most of the polyunsaturated fatty acids (PUFAs) at sn-2 of plasmalogens are docosahexaenoic acid (DHA; C22:6 n-3) or arachidonic acid (AA; C20:4 n-6) in animals (Nagan and Zoeller, 2001). The representative plasmalogens in mammalian tissues are plasmalogen phosphatidylethanolamine (PlsEtn) and phosphatidylcholine plasmalogen (PlsCho) 
(Heymans et al., 1983), and a small portion is present as plasmenylserine (PlsSer) and phosphatidic acid plasmalogen (Deeley et al., 2009; Ivanova et al., 2010; Nagy et al., 2012). PlsEtns constitute up to $50 \%$ of ethanolamine containing glycerophospholipids in the brain (Maeba et al., 2018).

Plasmalogens evolved first in anaerobic bacteria, but they are absent in facultative and aerobic bacteria except for Myxobacteria, which were recently found to use an oxygendependent synthetic pathway (Lorenzen et al., 2014; GallegoGarcía et al., 2019). Plasmalogens are not found in fungi or plants (Goldfine, 2010). It has been proposed that plasmalogen biosynthesis requiring molecular oxygen appeared later in animal cells as respiration evolved, indicating the emergence, disappearance and recurrence of plasmalogens during evolution. This disrupted evolution of plasmalogen may be due to the sensitivity of plasmalogens to reactive oxygen species (ROS) after the concentration of oxygen increased in the early earth's history, which will cause rapid degradation at the vinyl ether bond. However, the ability of higher organisms to use plasmalogen in an advantageous manner with special features of plasmalogens as found in animals, including antioxidant capacity, intracellular signaling and preservation of transmembrane ion gradients, may account for their reappearance (Goldfine, 2010; Lorenzen et al., 2014).

Lipid metabolism abnormalities are related to the occurrence of many human diseases (Fhaner et al., 2012). Plasmalogens are highly expressed in the nervous system and play an important role in many cellular functions of neurons (Maeba et al., 2018). Defects in plasmalogen synthesis are associated with neurodegenerative and metabolic diseases, such as Zellweger syndrome, Alzheimer's disease (AD), and Parkinson's disease (PD) (Yamashita et al., 2017; Dean and Lodhi, 2018). Among them, $\mathrm{AD}$ is an age-related progressive neurodegenerative disease and the cause of common dementia symptoms. The number of $\mathrm{AD}$ patients might reach more than 74 million worldwide by 2030, while the pathogeny of $\mathrm{AD}$ remains unclear (World Alzheimer Report, 2015). Plasmalogens were considered to be one of the oxidation targets of AD (Wood et al., 2010). The level of plasmalogens in blood and cerebrospinal fluid of $\mathrm{AD}$ patients is decreased (Goodenowe et al., 2007), and serum PlsEtn was suggested to be one of the cognitive decline markers (Maeba et al., 2018). In recent years, increasing studies demonstrated that supplemental of plasmalogens can be used to treat the symptoms of $\mathrm{AD}$ patients. Patients with mild $\mathrm{AD}$ showed a significant decrease in plasma PlsEtn in the placebo group than in the treatment group with oral administration of plasmalogens, and plasmalogens may improve cognitive functions of mild AD (Fujino et al., 2017). Moreover, serum plasmalogen levels have been used to diagnose and successfully stratify AD patients (Wood et al., 2015). These illustrate the importance of comprehensive understanding of the functions and biosynthesis of plasmalogens, which might be developed as a potential medicine for $\mathrm{AD}$.

This review summarized the enzymes (genes) and steps involved in the aerobic and anaerobic pathways of plasmalogens biosynthesis. The significance of recently found important genes and strategies for increasing the production and application potential of plasmalogens in medicine are discussed.

\section{PLASMALOGENS BIOSYNTHESIS IN ANAEROBIC BACTERIA}

The biosynthesis of plasmalogens differs in synthetic enzymes (genes) and substrates between anaerobic microorganisms and animals. In anaerobic bacteria, glycerol 3-phosphate has been confirmed as the precursor for plasmalogen synthesis (Hill and Lands, 1970; Prins and Van Golde, 1976), while dihydroxyacetone phosphate (DHAP) is the precursor of plasmalogens in animals. The enzymes related to phospholipid and plasmalogens synthesis identified in anaerobic bacteria up to date are listed in Table 1.

By measuring the kinetics of incorporation of ${ }^{32} \mathrm{Pi}$ and ${ }^{14} \mathrm{C}$ into the diacylphosphatides and plasmalogens using radioautography, the reaction steps of anaerobic pathway were investigated. In Clostridium beijerinckii ATCC 6015, rapid incorporation of ${ }^{32} \mathrm{Pi}$ into diacylphosphatidylethanolamine (diacyl-PtdEtn) and diacyl N-monomethyl PtdEtn, and a delayed incorporation into their corresponding plasmalogens, indicating that diacylphosphatide could be substrates for the corresponding plasmalogens (Baumann et al., 1965). A subsequent ${ }^{14} \mathrm{C}$-labeled acetate incorporation study also demonstrated a consistent precursor-product relationship between the chains attached to the phosphatidyl and alkyl-1-alkenyl ethers (Hagen and Goldfine, 1967). Moreover, labeling of the plasmalogen forms of phosphatidylglycerol (PtdGro) and cardiolipin is also delayed relative to the labeling of all acyl forms in C. beijerinckii (Koga and Goldfine, 1984). When hydroxylamine was added to the medium to block the decarboxylation of phosphatidylserine (PtdSer), there was initially $95 \%$ diacyl form and a 5\% plasmalogen form of PtdSer; PtdSer was rapidly decarboxylated to form PtdEtn followed by the PlsEtn after the removal of hydroxylamine (Goldfine, 2017).

A pathway of plasmalogen synthesis in anaerobic bacteria was proposed as shown in Figure 2 (Raetz and Dowhan, 1990; Dowhan, 1997; Zhang and Rock, 2008; Goldfine, 2010, 2017). First, fatty acyl-carrier protein (ACP) and glycerol 3phosphate serve as precursors of phosphate acid (PA) under the catalysis of PlsX and PlsY. PA with cytidine triphosphate (CTP) is converted to cytidine diphosphate diacylglycerol (CDPDAG) using CDP-diacylglycerol synthase (CdsA). Next, two additional transformations are required to produce PtdEtn or PtdGro. For PtdEtn synthesis, CDP-DAG can be converted to phosphatidylserine (PtdSer) using PtdSer synthase (PssA), and then PtdSer is converted to PtdEtn by PtdSer decarboxylase (Psd). For the branch of PtdGro synthesis, CDP-DAG is converted to phosphatidylglycerol 3-phosphate (PGP) by PGP synthase (PgsA) and then the 3-phosphate can be removed by a PGP phosphatase (PgpA or PgpB) to generate PtdGro (Dowhan, 1997). Finally, PtdEtn and PtdGro will be transformed into PlsEtn and PlsGro, respectively, under the catalysis of unknown enzymes. Although plasmalogens have been 




Diacyl glycerophospholipid

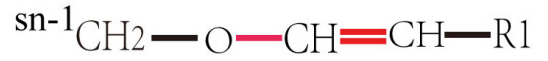<smiles>[R2]C(=O)O[C@H](C)[SnH3]</smiles><smiles>[X]OP(=O)(O)O[C@H](C)[SnH3]</smiles>

Plasmenyl glycerophospholipids (Plasmalogen)

FIGURE 1 | Structures of diacyl glycerophospholipid and plasmenyl glycerophospholipids (plasmalogens). X denotes the polar head group, such as ethanolamine or choline. R1 and R2 denote the hydrocarbon chains at the sn-1 and the sn-2 positions, respectively.

TABLE 1 | Enzymes related to plasmalogens biosynthesis in anaerobic bacteria.

\begin{tabular}{ll}
\hline Enzyme name & Function descriptions \\
\hline PlsXY & Glycerol 3-phosphate acyltransferase \\
CdsA & CDP-diacylglycerol synthase \\
PgsA & Phosphatidylglycerol phosphate synthase \\
PssA & Phosphatidylserine synthase \\
Psd & Phosphatidylserine decarboxylase \\
PgpA/PgpB & PGP phosphatases
\end{tabular}

CDP, cytidine diphosphate; PGP, phosphatidylglycerol phosphate.

identified in anaerobic bacteria for nearly 50 years (Wegner and Foster, 1963; Goldfine, 1964), gene(s) and mechanism corresponding to the formation of the vinyl ether bond of plasmalogens remain unclear.

\section{THE OXYGEN-DEPENDENT PATHWAY OF PLASMALOGENS BIOSYNTHESIS}

Phosphatidylethanolamine plasmalogen is the basic components of cell bilayers, accounting for about $20 \%$ of human phospholipids (Farooqui and Horrocks, 2001; Lessig and Fuchs, 2009; Braverman and Moser, 2012; Dorninger et al., 2015; Dean and Lodhi, 2018). Particularly, high concentrations of PlsEtn were found in the brain, retina, and other nervous tissues, accounting for $60 \%$ and $80 \%$ of the total ethanolamine phospholipids in gray and white matter, respectively (Saab et al., 2014). The plasmalogen-associated genes in animals have been studied as listed in Table 2. Among them, plasmanyl desaturase $\left(1^{\prime}\right.$-alkyl desaturase) is a predicted unstable membrane enzyme that remains to be identified for many years. In a recent report, the enzyme CarF found in an aerobic bacterium Myxobacteria

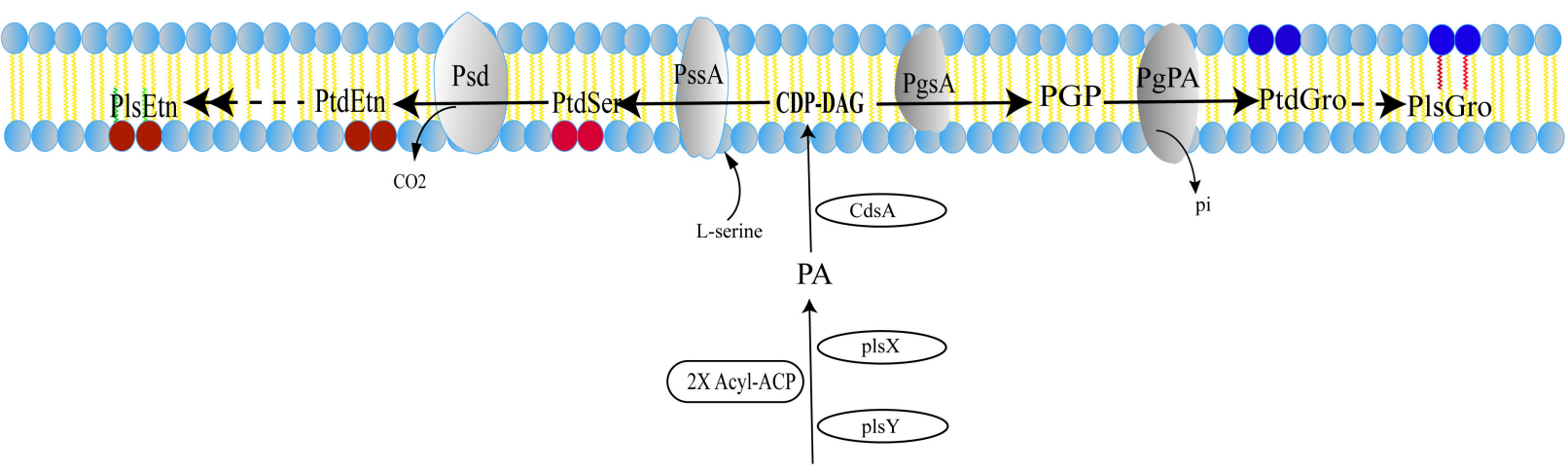

Glycerol-p

FIGURE 2 | Anaerobic pathway for plasmalogen synthesis in bacteria. PA, phosphatidic acid; CDP-DAG, CDP-diacylglycerol; PGP, phosphatidylglycerol 3-phosphate; PtdEtn, phosphatidylethanolamine; PtdSer, phosphatidylserine; PtdGro, phosphatidylglycerol; PlsGro, phosphatidylglycerol plasmalogen; PlsEtn, phosphatidylethanolamine plasmalogen. The detail of enzyme and mechanism leading from the diacyl phospholipids to plasmalogens is still unknown. 
TABLE 2 | Enzymes related to plasmalogen biosynthesis in animals.

\begin{tabular}{ll}
\hline Enzyme name & Function descriptions \\
\hline FAS & Fatty acid synthase \\
ACS & Acyl-CoA synthase \\
FAR-1/2 & Fatty acyl-CoA reductase 1 or 2 \\
DHAP-AT & DHAP acyltransferase \\
ADHAP-S & Alkyl DHAP synthase \\
AADHAP-R & Alkyl/acyl-DHAP-reductase \\
AAG3P-AT & Alkyl/acyl-glycero-3-phosphate acyltransferase \\
PH & Phosphohydrolase \\
E-PT & Ethanolamine phosphotransferase \\
TMEM189 homolog & plasmanylethanolamine desaturase
\end{tabular}

DHAP, dihydroxyacetone phosphate.

was confirmed to be the $1^{\prime}$-alkyl desaturase for the last step of plasmalogen formation (Gallego-García et al., 2019). Its homolog TMEM189 was successively identified in mice and human (Gallego-García et al., 2019; Werner et al., 2020). Knock out of the animal homolog in human cell lines resulted in the deficiency of plasmalogens, indicating that TMEM189 is needed to catalyze the final step in plasmalogen synthesis in human cells (Gallego-García et al., 2019).

Plasmalogen biosynthesis occurs in peroxisomes, an oxidative organelle found in virtually all eukaryotic cells, and terminates in the endoplasmic reticulum (ER) (Wallner and Schmitz,
2011). Based on the identified enzymes in previous publications (Wallner and Schmitz, 2011; Gallego-García et al., 2019; Werner et al., 2020), an overview of the synthetic pathway is proposed in Figure 3.

Plasmalogens biosynthesis in peroxisomes of animals starts with acyl-CoA and DHAP. Under the action of DHAPacyltransferase (DHAP-AT), DHAP is converted into 1-Oacyl DHAP, and then the acyl chain is replaced by a long-chain fatty alcohol from Acyl-CoA synthesis pathway (McIntyre et al., 2008) or from foods. DHAP-acyltransferase is also known as glyceronephosphate $O$-acyltransferase (GNPAT) (Nagan and Zoeller, 2001), which initiates the esterification of DHAP with a long-chain acyl-CoA (Hajra, 1997). Next, alkyl DHAP synthase (ADHAP-S) catalyzes the replacement of acyl group of 1-O-acyl DHAP with a long-chain fatty alcohol to generate 1-O alkyl-DHAP (Hajra, 1995; Hayashi and Sato, 1997; Cheng and Russell, 2004; Honsho et al., 2010; Wallner and Schmitz, 2011). The fatty alcohols are synthesized by fatty acyl-CoA reductases 1 and 2 (Far1/2) (Hajra, 1995, 1997; Nagan and Zoeller, 2001; Wallner and Schmitz, 2011) or directly taken up from diet. Notably, the Farl is regulated by negative feedback of cellular plasmalogen levels (Honsho et al., 2010). Therefore, the formation and supply of longchain fatty alcohols are considered to be one of the ratelimiting steps of the plasmalogen biosynthetic pathway (Paul et al., 2019). The alkyl-DHAP is then transferred from the peroxisome into the ER.

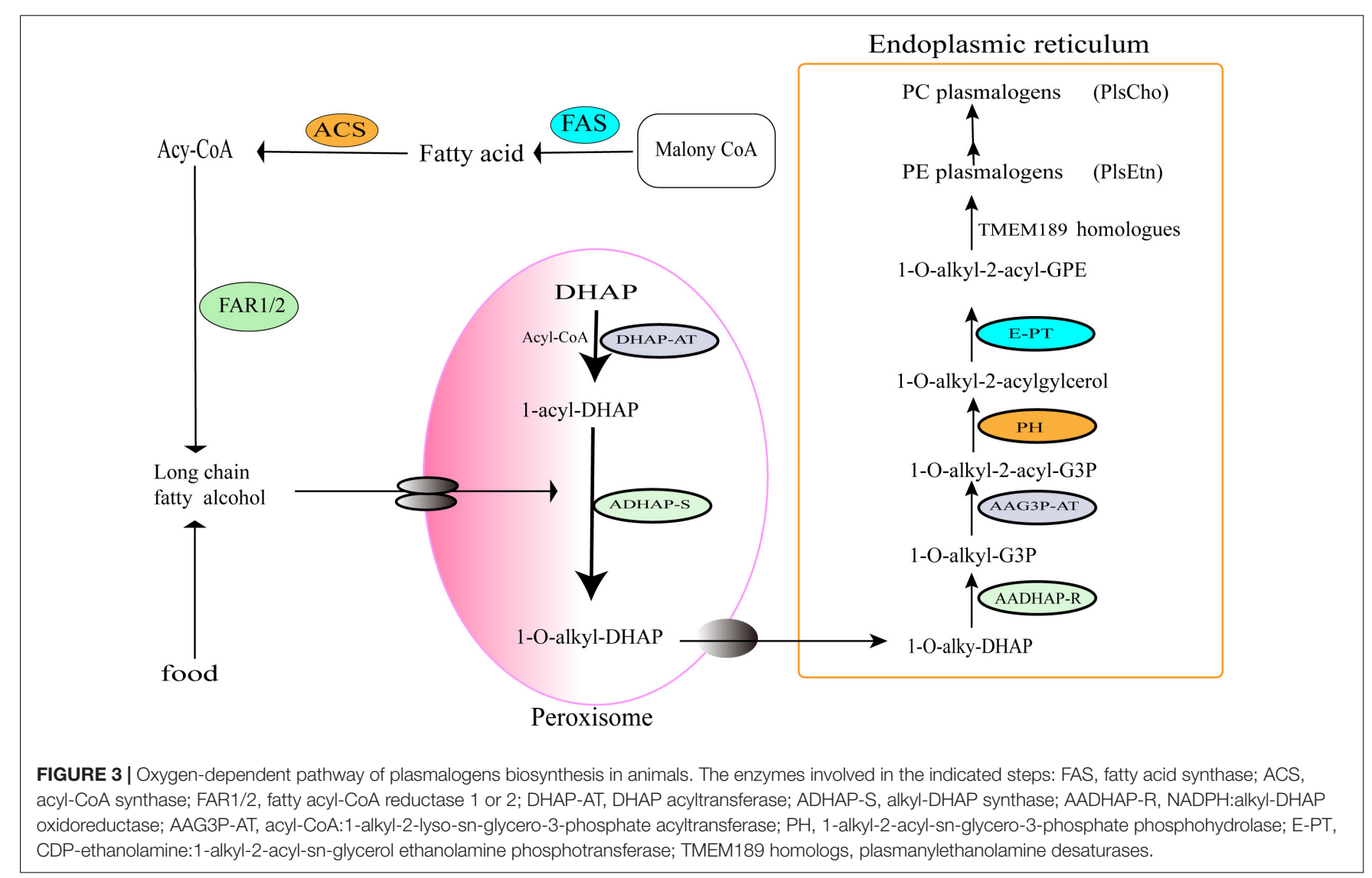


In the ER, acyl/alkyl DHAP reductase (AADHAP-R) catalyzes the reduction of 1-O-alkyl-DHAP to form 1-O-alkyl-2-hydroxylsn-glycerol-3-phosphate (1-O-alkyl-G3P) (Hajra, 1997). Then, the acyl/alkyl-G3P-acyltransferase (AAG3P-AT) catalyzes acylation with an acyl-CoA at the sn-2 position of the 1-Oalkyl-G3P to form 1-O-alkyl-2-acyl-G3P. The phosphate group of -O-alkyl-2-acyl-G3P is removed by phosphohydrolase $(\mathrm{PH})$ to form 1-O-alkyl-2-acylglycerol (Wallner and Schmitz, 2011). Next, ethanolamine phosphate head group is added to 1-Oalkyl-2-acylglycerol by the ethanolamine phosphotransferase (E-PT) to generate plasmanylethanolamine (1-O-alkyl-2-acylGPE) (Brites et al., 2004). Finally, plasmanylethanolamine is converted into 1 -O-alk-1' -enyl 2 -acyl phosphatidylethanolamine (PlsEtn) through the action of $1^{\prime}$-alkyl desaturase (TMEM189 homolog) in the presence of molecular oxygen and NADPH. Because there is no plasmenylcholine desaturase found in animals, choline plasmalogens (PlsCho) might be formed only following the hydrolysis of ethanolamine plasmalogens into form 1-O -(1Z-alkenyl)-2-acyl-sn-glycerol, which was then modified by choline phosphotransferase and CDP-choline (Lee, 1998).

\section{PERSPECTIVES OF PLASMALOGENS BIOSYNTHESIS USING SYNTHETIC BIOLOGY METHODS AND APPLICATION POTENTIALS IN MEDICINE}

Traditionally, plasmalogens are obtained using chemical synthetic method or extraction from animal tissue. However, the need for large amounts of chemicals as well as generation of potential hazardous waste during the chemical synthetic process of plasmalogens limit the applications of the chemical synthetic method. Although plasmalogens are widely found and can be prepared from marine animals or bird tissues, the amount of plasmalogens from these natural materials are very low and only account for less than $10 \%$ of the phospholipids in cell membrane of the tissues used.

Synthetic biology is a discipline that uses biological functional elements, devices and systems to carry out targeted genetic design and transformation of living organisms, to enable cells and organisms to generate specific biological functions or produce natural materials and even to synthesize "artificial life." Using the synthetic biology techniques, artificial PUFA biosynthetic gene cluster (BGC) including a polyketide synthase-like PUFA synthetase from Myxobacteria has been introduced into yeast Yarrowia lipolytica, and successfully produces the highest level of DHA (16.8\% of total fatty acid) among PUFA-producing Y. lipolytica (Gemperlein et al., 2019).

With the elucidation of plasmalogen biosynthesis genes and pathway for aerobic organisms, especially the recent identification of $1^{\prime}$-alkyl desaturase responsible for the conversion of plasmanylphospholipid into plasmalogens (Gallego-García et al., 2019), designing and efficient expression of plasmalogen biosynthetic modules in engineering host cells such as yeast cells become possible. The production and composition of plasmalogens is controlled by synthetic genes and certain rate-limiting steps in biosynthesis such as
Far1/2 and 1-alkyl-DHAP (Paul et al., 2019). It was found that supplementation with alkyl glycerol can increase plasmalogen levels in cultured cells (Marigny et al., 2002), animals (Brites et al., 2011), and humans (Das et al., 1992). The most commonly used alkyl glycerols to increase plasmalogen levels in mammalian research are chimyl (O-16:0), batyl (O-18:0), and selachyl (O-18:1) alcohols (Brites et al., 2011; Rasmiena et al., 2015; Tham et al., 2018). Therefore, expression of plasmalogen products can be regulated under specific conditions through the genetic circuit designing and integration of gene modules composed of plasmalogen-related genes and rate-limiting elements. Standard and modularized biological elements can be used to reconstruct the metabolic network in host cells to efficiently synthesize or improve plasmalogen products that meet needs. For anaerobic biosynthesis of plasmalogen, it is necessary for us to identify the key gene(s) responsible for the formation of the vinyl ether bond of plasmalogens in anaerobic bacteria before its synthetic biological study and application.

It has been known that cultured cells and animal tissues lacking plasmalogen are more sensitive to oxidative damage than their wild-type counterparts (Zoeller et al., 1988; Reiss et al., 1997). This is due to the presence of vinyl ether bonds making plasmalogens efficient antioxidants (Broniec et al., 2011). In particular, plasmalogens can protect unsaturated membrane lipids from oxidation by singlet oxygen and participate in the removal of various ROS (Maeba et al., 2002; Skaff et al., 2008). Plasmalogen is susceptible to cleavage by ROS, yielding products that may act as second messengers (Lorenzen et al., 2014). More importantly, plasmalogen deficiency correlates with various human neurological and aging diseases, such as $\mathrm{AD}$ and PD (Nadeau et al., 2019; Paul et al., 2019).

Alzheimer's disease is a complex neurodegenerative disease characterized by progressive memory loss and progressive loss of neuronal cells mainly observed in the hippocampus (Fujino et al., 2017; Jan et al., 2017). Although the gradual accumulation of $\beta$-amyloid fibers (A $\beta$ plaque) and abnormal forms of tau (tau tangles) inside and outside neurons are considered the neuropathology of $\mathrm{AD}$, the causes and mechanisms of $\mathrm{AD}$ have not been fully elucidated (Fujino et al., 2017; Jan et al., 2017). Accumulation of $\beta$-amyloid in $\mathrm{AD}$ leads to the increase of ROS levels in cells and reduces the activity of ADHAP-S, which might result in the decrease of plasmalogens (Grimm et al., 2011). Plasmalogen levels in human serum decrease with age and reductions in alkyl PtdCho and alkyl PtdEtn levels have been observed in patients with hypertension (Graessler et al., 2009). The content of plasmalogens in the brain of $\mathrm{AD}$ patients after death is very low (Wood et al., 2010; Braverman and Moser, 2012). Among them, the PlsEtn decreased by about 70\%(Wood et al., 2010; Onodera et al., 2015). Hossain et al. (2013, 2016) found that PlsEtn inhibited the death of hippocampal neurons by increasing the phosphorylation of Akt and ERK kinases through activating the neuronal specific orphan G-protein coupled receptors (GPCRs). In their study, pan GPCR inhibitors significantly reduce the plasmalogens-induced ERK signaling in nerve cells, indicating that plasmalogens could activate GPCRinduced signaling, Plasmalogens-mediated phosphorylation of ERK was inhibited in five of the GPCRs' knockdown cells. Overexpression of these GPCRs enhanced the plasmalogens- 
mediated phosphorylation of ERK and Akt, and the GPCRsmediated cellular signaling was reduced significantly when the endogenous plasmalogens were reduced, suggesting for the first time a possible mechanism of plasmalogens-induced cell signaling in the nervous system (Hossain et al., 2016). Direct consumption of plasmalogen or related phospholipids can be used to treat dementia. For example, DHA-PC and DHA-PS can restore the content of DHA-containing PS and PlsEtn in the brain, and significantly restore the lipid homeostasis of dementia mice (SAMP8 mice), which have a phenotype that accelerates aging (Zhao et al., 2020). Oral administration of PtdEtn rich in plasmalogens (PlsEtn) from viscera of marine animals ameliorated cognitive impairment and improved the learning ability in amyloid (A $\beta$ )-infused rats (Yamashita et al., 2017). In recent human trials, Fujino et al. (2017) reported that oral supplementing scallop-derived purified plasmalogens ( $1 \mathrm{mg} /$ day) for 24 weeks improved memory function of patients with mild AD. Hossain et al. (2018) reported that oral ingestion of plasmalogens can attenuate the lipopolysaccharide-induced memory loss and microglial activation in mice. These findings suggest the importance of comprehensive understanding of the functions and biosynthesis of plasmalogens, which might be developed as a potential medicine for AD. Due to the increasing need of plasmalogens, it is possible to biosynthesize plasmalogens on a large scale using synthetic biological strategy.

Parkinson's disease is a metabolic disorder and neurodegenerative disease. The pathological feature is the abnormal aggregation of SNCA/ $\alpha$-synuclein in the brain and the loss of dopaminergic neurons in the substantia nigra (Ho et al., 2020). The relationship between PD and plasmalogen was controversial. Although the initial study found no changes in the PlsEtn of PD patients compared with the control group (Ginsberg et al., 1995), recent studies have found that the serum concentration of PlsEtn in PD patients is reduced and low levels of plasmalogen have also been detected in frontal lobe sebaceous rafts of PD patients (Dragonas et al., 2009; Fabelo et al., 2011). Nadeau et al. (2019) reported the neuroprotective and immunomodulatory effects of plasmalogen precursors on mice with PD. They found that the supplement of DHA-containing PlsEtn precursor PPI-1011 in the intestine of mice treated with 1methyl-4-phenyl-1,2,3,6- tetrahydropyridine (MPTP) could not only prevent MPTP-induced decrease in PlsEtn levels but also

\section{REFERENCES}

Baumann, N. A., Hagen, P. O., and Goldfine, H. (1965). Phospholipids of Clostridium butyricum. studies on plasmalogen composition and biosynthesis. J. Biol. Chem. 240, 1559-1567.

Braverman, N. E., and Moser, A. B. (2012). Functions of plasmalogen lipids in health and disease. Biochim. Biophys. Acta 1822, 1442-1452. doi: 10.1016/j. bbadis.2012.05.008

Brites, P., Ferreira, A. S., da Silva, T. F., Sousa, V. F., Malheiro, A. R., Duran, M., et al. (2011). Alkyl-glycerol rescues plasmalogen levels and pathology of etherphospholipid deficient mice. PLoS One 6:e28539. doi: 10.1371/journal.pone. 0028539

Brites, P., Waterham, H. R., and Wanders, R. J. (2004). Functions and biosynthesis of plasmalogens in health and disease. Biochim. Biophys. Acta 1636, 219-231. doi: 10.1016/j.bbalip.2003.12.010 reduce macrophage infiltration in the intermuscular plexus of MPTP-treated mice (Nadeau et al., 2019). These results indicate the potential application of PlsEtn in the treatment of PD.

\section{CONCLUSION}

This review summarizes the current knowledge in the field of anaerobic and aerobic biosynthetic pathways and application potential of plasmalogens in medicine. The anaerobic biosynthesis of plasmalogens differs in synthetic genes and precursors from that of oxygen-dependent biosynthesis pathway. Two different biosynthetic pathways demonstrate the significant functions and evolution of plasmalogens in organisms. The recent identification of $1^{\prime}$-alkyl desaturase elucidated the aerobic plasmalogen biosynthesis pathway and opened the door to the aerobic synthesis of plasmalogens using synthetic biological strategy. Further investigation on the genes responsible for the critical step in anaerobic synthesis pathway is required for the comprehensive understanding of plasmalogens evolution and functions. Because of the relevance of plasmalogens to neurological diseases, it is increasingly important to investigate the production and application of plasmalogens as potential therapeutic strategies for treating and preventing neurodegenerative and metabolic diseases.

\section{AUTHOR CONTRIBUTIONS}

BT and YZ conceived the review and wrote the manuscript. NY, JZ, ZX, and ZY revised the literature and helped to writing the manuscript. BT supervised the overall project and edited the manuscript. All authors had the opportunity to discuss and comment on the manuscript.

\section{FUNDING}

This study was supported by grants from the National Natural Science Foundation of China (31670083 and 31870025), the National Key R\&D Program of China (2019YFA0905400), and the Fundamental Research Funds for the Central Universities.

Broniec, A., Klosinski, R., Pawlak, A., Wrona-Krol, M., Thompson, D., and Sarna, T. (2011). Interactions of plasmalogens and their diacyl analogs with singlet oxygen in selected model systems. Free Radic Biol. Med. 50, 892-898. doi: 10.1016/j.freeradbiomed.2011.01.002

Cheng, J. B., and Russell, D. W. (2004). Mammalian wax biosynthesis I. Identifification of two fatty acyl-Coenzyme A reductases with different substrate specifificities and tissue distributions. J. Biol. Chem. 279, 37789-37797. doi: 10.1074/jbc.m406225200

Das, A. K., Holmes, R. D., Wilson, G. N., and Hajra, A. K. (1992). Dietary ether lipid incorporation into tissue plasmalogens of humans and rodents. Lipids 27, 401-405. doi: 10.1007/bf02536379

Dean, J. M., and Lodhi, I. J. (2018). Structural and functional roles of ether lipids. Protein Cell 9, 196-206. doi: 10.1007/s13238-017-0423-5

Deeley, J. M., Thomas, M. C., Truscott, R. J., Mitchell, T. W., and Blanksby, S. J. (2009). Identification of abundant alkyl ether glycerophospholipids in 
the human lens by tandem mass spectrometry techniques. Anal. Chem. 81, 1920-1930. doi: 10.1021/ac802395d

Dorninger, F., Brodde, A., Braverman, N. E., Moser, A. B., Just, W. W., Forss-Petter, S., et al. (2015). Homeostasis of phospholipids - The level of phosphatidylethanolamine tightly adapts to changes in ethanolamine plasmalogens. Biochim. Biophys. Acta 1851, 117-128. doi: 10.1016/j.bbalip. 2014.11.005

Dowhan, W. (1997). Molecular basis for membrane phospholipid diversity: why are there so many lipids? Annu. Rev. Biochem. 66, 199-232. doi: 10.1146/ annurev.biochem.66.1.199

Dragonas, C., Bertsch, T., Sieber, C. C., and Brosche, T. (2009). Plasmalogens as a marker of elevated systemic oxidative stress in Parkinson's disease. Clin. Chem. Lab. Med. 47, 894-897.

Fabelo, N., Martín, V., Santpere, G., Marín, R., Torrent, L., Ferrer, I., et al. (2011). Severe alterations in lipid composition of frontal cortex lipid rafts from Parkinson's disease and incidental Parkinson's disease. Mol. Med. 17, 1107-1118. doi: 10.2119/molmed.2011.00119

Farooqui, A. A., and Horrocks, L. A. (2001). Plasmalogens: workhorse lipids of membranes innormal and injured neurons and glia. Neuroscientist 7, 232-245. doi: $10.1177 / 107385840100700308$

Ferlay, J., Soerjomataram, I., Dikshit, R., Eser, S., Mathers, C., Rebelo, M., et al. (2015). Cancer incidence and mortality worldwide: sources, methods and major patterns in GLOBOCAN 2012. Int. J. Cancer 136, E359-E386.

Fhaner, C. J., Liu, S., Ji, H., Simpson, R. J., and Reid, G. E. (2012). Comprehensive lipidome profiling of isogenic primary and metastatic colon adenocarcinoma cell lines. Anal. Chem. 84, 8917-8926. doi: 10.1021/ac302154g

Fujino, T., Yamada, T., Asada, T., Tsuboi, Y., Wakana, C., Mawatari, S., et al. (2017). Efficacy and blood plasmalogen changes by oral administration of plasmalogen in patients with mild Alzheimer's disease and mild cognitive impairment: a multicenter, randomized, double-blind, placebo-controlled trial. eBio Med. 17, 199-205. doi: 10.1016/j.ebiom.2017.02.012

Gallego-García, A., Monera-Girona, A. J., Pajares-Martínez, E., Bastida-Martínez, E., Pérez-Castaño, R., Iniesta, A. A., et al. (2019). Bacterial light response reveals an orphan desaturase for human plasmalogen synthesis. Science 366, 128-132. doi: 10.1126/science.aay1436

Gemperlein, K., Dietrich, D., Kohlstedt, M., Zipf, G., Bernauer, H. S., Wittmann, C., et al. (2019). Polyunsaturated fatty acid production by Yarrowia lipolytica employing designed myxobacterial PUFA synthases. Nat. Commun. 10:4055. doi: 10.1038/s41467-019-12025-8

Ginsberg, L., Rafique, S., Xuereb, J. H., Rapoport, S. I., and Gershfeld, N. L. (1995). Disease and anatomic specificity of ethanolamine plasmalogen deficiency in Alzheimer's disease brain. Brain Res. 698, 223-226. doi: 10.1016/0006-8993(95) 00931-f

Goldfine, H. (1964). Composition of the aldehydes of Clostridium butyricum plasmalogens: cyclopropane aldehydes. J. Biol. Chem. 239, 2130-2134.

Goldfine, H. (2010). The appearance, disappearance and reappearance of plasmalogens in evolution. Prog. Lipid Res. 49, 493-498. doi: 10.1016/j.plipres. 2010.07.003

Goldfine, H. (2017). The anaerobic biosynthesis of plasmalogens. FEBS Lett. 591, 2714-2719. doi: 10.1002/1873-3468.12714

Goodenowe, D. B., Cook, L. L., Liu, J., Lu, Y., Jayasinghe, D. A., Ahiahonu, P. W., et al. (2007). Peripheral ethanolamine plasmalogen deficiency: a logical causative factor in Alzheimer's disease and dementia. J. Lipid Res. 48, 24852498. doi: 10.1194/jlr.p700023-jlr200

Graessler, J., Schwudke, D., Schwarz, P. E., Herzog, R., Shevchenko, A., and Bornstein, S. R. (2009). Top-down lipidomics reveals ether lipid deficiency in blood plasma of hypertensive patients. PLoS One 15:e6261. doi: 10.1371/journal.pone. 006261

Grimm, M. O., Kuchenbecker, J., Rothhaar, T. L., Grösgen, S., Hundsdörfer, B., Burg, V. K., et al. (2011). Plasmalogen synthesis is regulated via alkyldihydroxyacetonephosphate-synthase by amyloid precursor protein processing and is affected in Alzheimer's disease. J. Neurochem. 116, 916-925. doi: 10.1111/ j.1471-4159.2010.07070.x

Hagen, P. O., and Goldfine, H. (1967). Phospholipids of Clostridium butyricum. 3. Further studies on the origin of the aldehyde chains of plasmalogens. J. Biol. Chem. 242, 5700-5708.

Hajra, A. K. (1995). Glycerolipid biosynthesis in peroxisomes (microbodies). Prog. Lipid Res. 34, 343-364. doi: 10.1016/0163-7827(95)00013-5
Hajra, A. K. (1997). Dihydroxyacetone phosphate acyltransferase. Biochim. Biophys. Acta 1348, 27-34.

Hayashi, H., and Sato, A. (1997). Fatty alcohol synthesis accompanied with chain elongation in liver peroxisomes. Biochim. Biophys. Acta 1346, 38-44. doi: 10.1016/s0005-2760(97)00020-9

Heymans, H. S. A., Schutgens, R. B. H., Tan, R., van den Bosch, H., and Borst, P. (1983). Severe plasmalogen defificiency in tissues of infants without peroxisomes (Zellweger syndrome). Nature 306, 69-70. doi: 10.1038/306069a0

Hill, E. E., and Lands, W. E. (1970). Formation of acyl and alkenyl glycerol derivatives in Clostridium butyricum. Biochim. Biophys. Acta 202, 209-211. doi: 10.1016/0005-2760(70)90239-0

Ho, P. W., Leung, C. T., Liu, H., Pang, S. Y. Y., Lam, C. S. C., Xian, J., et al. (2020). Age-dependent accumulation of oligomeric SNCA/ $\alpha$-synuclein from impaired degradation in mutant LRRK2 knockin mouse model of Parkinson disease: role for therapeutic activation of chaperone-mediated autophagy (CMA). Autophagy 16, 347-370. doi: 10.1080/15548627.2019.1603545

Honsho, M., Asaoku, S., and Fujiki, Y. (2010). Posttranslational regulation of fatty acyl-CoA reductase 1, Farl, controls ether glycerophospholipid synthesis. J. Biol. Chem. 285, 8537-8542. doi: 10.1074/jbc.m109.083311

Hossain, M. S., Ifuku, M., Take, S., Kawamura, J., Miake, K., and Katafuchi, T. (2013). Plasmalogens rescue neuronal cell death through an activation of AKT and ERK survival signaling. PLoS One 8:e83508. doi: 10.1371/journal.pone. 0083508

Hossain, M. S., Mineno, K., and Katafuchi, T. (2016). Neuronal orphan G-protein coupled receptor proteins mediate plasmalogens-induced activation of ERK and Akt signaling. PLoS One 11:e0150846. doi: 10.1371/journal.pone.00150846

Hossain, M. S., Tajima, A., Kotoura, S., and Katafuchi, T. (2018). Oral ingestion of plasmalogens can attenuate the LPS-induced memory loss and microglial activation. Biochem. Biophys. Res. Commun. 496, 1033-1039. doi: 10.1016/j. bbrc.2018.01.078

Ivanova, P. T., Milne, S. B., and Brown, H. A. (2010). Identification of atypical ether-linked glycerophospholipid species in macrophages by mass spectrometry. J. Lipid Res. 51, 1581-1590. doi: 10.1194/jlr. d 003715

Jan, A. T., Azam, M., Rahman, S., Almigeiti, A. M. S., Choi, D. H., Lee, E. J., et al. (2017). Perspective insights into disease progression, diagnostics, and therapeutic approaches in Alzheimer's disease: a judicious update. Front. Aging Neurosci. 9:356. doi: 10.3389/fnagi.2017.00356

Koga, Y., and Goldfine, H. (1984). Biosynthesis of phospholipids in Clostridium butyricum: kinetics of synthesis of plasmalogens and the glycerol acetal of ethanolamine plasmalogen. J. Bacteriol. 159, 597-604. doi: 10.1128/jb.159.2. 597-604.1984

Lee, T. C. (1998). Biosynthesis and possible biological functions of plasmalogens. Biochim. Biophys. Acta 1394, 129-145. doi: 10.1016/s0005-2760(98)00107-6

Lessig, J., and Fuchs, B. (2009). Plasmalogens in biological systems: their role in oxidative processes in biological membranes, their contribution to pathological processes and aging and plasmalogen analysis. Curr. Med. Chem. 16, 20212041. doi: 10.2174/092986709788682164

Lorenzen, W., Ahrendt, T., Bozhüyük, K. A., and Bode, H. B. (2014). A multifunctional enzyme is involved in bacterial ether lipid biosynthesis. Nat. Chem. Biol. 10, 425-427. doi: 10.1038/nchembio.1526

Maeba, R., Araki, A., and Fujiwara, Y. (2018). Serum ethanolamine plasmalogen and urine myo-inositol as cognitive decline markers. Adv. Clin. Chem. 87, 69-111. doi: 10.1016/bs.acc.2018.08.001

Maeba, R., Sawada, Y., Shimasaki, H., Takahashi, I., and Uetaa, N. (2002). Ethanolamine plasmalogens protect cholesterol-rich liposomal membranes from oxidation caused by free radicals. Chem. Phys. Lipids 120, 145-151. doi: 10.1016/s0009-3084(02)00101-9

Marigny, K., Pedrono, F., Martin-Chouly, C. A., Youmine, H., Saiag, B., and Legrand, A. B. (2002). Modulation of endothelial permeability by 1-Oalkylglycerols. Acta Physiol. Scand. 176, 263-268. doi: 10.1046/j.1365-201x. 2002.01037.x

McIntyre, T. M., Snyder, F., and Marathe, G. K. (2008). "Ether-linked lipids and their bioactive species," in Biochemistry of Lipids, Lipoproteins and Membranes, eds D. E. Vance and J. E. Vance (Amsterdam: Elsevier), 245-276. doi: 10.1016/ b978-044453219-0.50011-8

Nadeau, J., Smith, T., Lamontagne-Proulx, J., Bourque, M., Al Sweidi, S., Jayasinghe, D., et al. (2019). Neuroprotection and immunomodulation in the 
gut of parkinsonian mice with a plasmalogen precursor. Brain Res. 1725:146460. doi: 10.1016/j.brainres.2019.146460

Nagan, N., and Zoeller, R. A. (2001). Plasmalogens: biosynthesis and functions. Prog. Lipid Res. 40, 199-229. doi: 10.1016/s0163-7827(01)00003-0

Nagy, K., Brahmbhatt, V. V., Berdeaux, O., Bretillon, L., Destaillats, F., and Acar, N. (2012). Comparative study of serine-plasmalogens in human retina and optic nerve: identification of atypical species with odd carbon chains. J. Lipid Res. 53, 776-783. doi: 10.1194/jlr.d022962

Onodera, T., Futai, E., Kan, E., Abe, N., Uchida, T., Kamio, Y., et al. (2015). Phosphatidylethanolamine plasmalogen enhances the inhibiting effect of phosphatidylethanolamine on $\gamma$-secretase activity. J. Biochem. 157, 301-309. doi: $10.1093 / \mathrm{jb} / \mathrm{mvu} 074$

Paul, S., Lancaster, G. I., and Meikle, P. J. (2019). Plasmalogens: a potential therapeutic target for neurodegenerative and cardiometabolic disease. Prog. Lipid Res. 74, 186-195. doi: 10.1016/j.plipres.2019.04.003

Prins, R. A., and Van Golde, L. M. (1976). Entrance of glycerol into plasmalogens of somestrictly anaerobic bacteria and protozoa. FEBS Lett. 63, 107-111. doi: 10.1016/0014-5793(76)80204-9

Raetz, C. R., and Dowhan, W. (1990). Biosynthesis and function of phospholipids in Escherichia coli. J. Biol. Chem. 265, 1235-1238.

Rasmiena, A. A., Barlow, C. K., Stefanovic, N., Huynh, K., Tan, R., Sharma, A., et al. (2015). Plasmalogen modulation attenuates atherosclerosis in ApoEand ApoE/GPx1-deficient mice. Atherosclerosis 243, 598-608. doi: 10.1016/j. atherosclerosis.2015.10.096

Reiss, D., Beyer, K., and Engelmann, B. (1997). Delayed oxidative degra dation of polyunsaturated diacyl phospholipids in the presence of plasmalogen phospholipids in vitro. Biochem. J. 323, 807-814. doi: 10.1042/bj3230807

Saab, S., Mazzocco, J., Creuzot-Garcher, C. P., Bron, A. M., Bretillon, L., and Acar, N. (2014). Plasmalogens in the retina: from occurrence in retinal cell membranes to potential involvement in pathophysiology of retinal diseases. Biochimie 107(Pt A), 58-65. doi: 10.1016/j.biochi.2014.07.023

Skaff, O., Pattison, D. I., and Davies, M. J. (2008). The vinyl ether linkages of plasmalogens are favored targets for myeloperoxidase-derived oxidants: a kinetic study. Biochemistry 47, 8237-8245. doi: 10.1021/bi800786q

Snyder, F. (1999). The ether lipid trail: a historical perspective. Biochim. Biophys. Acta 1436, 265-278.

Tham, Y. K., Huynh, K., Mellett, N. A., Henstridge, D. C., Kiriazis, H., Ooi, J. Y. Y., et al. (2018). Distinct lipidomic profiles in models of physiological and pathological cardiac remodeling, and potential therapeutic strategies. Biochim. Biophys. Acta Mol. Cell Biol. Lipids 1863, 219-234. doi: 10.1016/j.bbalip.2017. 12.003

Wallner, S., and Schmitz, G. (2011). Plasmalogens the neglected regulatory and scavenging lipid species. Chem. Phys. Lipids 164, 573-589. doi: 10.1016/j. chemphyslip.2011.06.008
Wegner, G. H., and Foster, E. M. (1963). Incorporation of isobutyrate and valerate into cellular plasmalogen by Bacteroides succinogenes. J. Bacteriol. 85, 53-61. doi: $10.1128 / \mathrm{jb} .85 .1 .53-61.1963$

Werner, E. R., Keller, M. A., Sailer, S., Lackner, K., Koch, J., Hermann, M., et al. (2020). The TMEM189 gene encodes plasmanylethanolamine desaturase which introduces the characteristic vinyl ether double bond into plasmalogens. Proc. Natl. Acad. Sci. U.S.A. 117, 7792-7798. doi: 10.1073/pnas.1917461117

Wood, P. L., Locke, V. A., Herling, P., Passaro, A., Vigna, G. B., Volpato, S., et al. (2015). Targeted lipidomics distinguishes patient subgroups in mild cognitive impairment (MCI) and late onset Alzheimer's disease (LOAD). BBA Clin. 5, 25-28. doi: 10.1016/j.bbacli.2015.11.004

Wood, P. L., Mankidy, R., Ritchie, S., Heath, D., Wood, J. A., Flax, J., et al. (2010). Circulating plasmalogen levels and Alzheimer Disease Assessment Scale-Cognitive scores in Alzheimer patients. J. Psychiatry Neurosci. 35, 59-62. doi: 10.1503/jpn.090059

World Alzheimer Report (2015). The Global Impact of Dementia: An Analysis of Prevalence, Incidence, Cost and Trends. London: Alzheimer's Disease International.

Yamashita, S., Hashimoto, M., Haque, A. M., Nakagawa, K., Kinoshita, M., Shido, Q., et al. (2017). Oral administration of ethanolamine glycerophospholipid containing a high level of plasmalogen improves memory impairment in myloid $\beta$-infused rats. Lipids 52, 575-585. doi: 10.1007/s11745-0174260-3

Zhang, Y. M., and Rock, C. O. (2008). Membrane lipid homeostasis in bacteria. Nat. Rev. Microbiol. 6, 222-233. doi: 10.1038/nrmicro1839

Zhao, Y. C., Zhou, M. M., Zhang, L. Y., Cong, P. X., Xu, J., Xue, C. H., et al. (2020). Recovery of brain DHA-containing phosphatidylserine and ethanolamine plasmalogen after dietary DHA-enriched phosphatidylcholine and phosphatidylserine in SAMP8 mice fed with high-fat diet. Lipids Health Dis. 19:104.

Zoeller, R. A., Morand, O. H., and Raetz, C. R. (1988). A possible role for plasmalogens in protecting animal cells against photosensitized killing. J. Biol. Chem. 263, 11590-11596.

Conflict of Interest: The authors declare that the research was conducted in the absence of any commercial or financial relationships that could be construed as a potential conflict of interest.

Copyright (C) 2020 Zhou, Yu, Zhao, Xie, Yang and Tian. This is an open-access article distributed under the terms of the Creative Commons Attribution License (CC BY). The use, distribution or reproduction in other forums is permitted, provided the original author(s) and the copyright owner(s) are credited and that the original publication in this journal is cited, in accordance with accepted academic practice. No use, distribution or reproduction is permitted which does not comply with these terms. 